\title{
Tempo de Graduação em Medicina: uma Estimativa em 15 Coortes de Graduados na Universidade Agostinho Neto, Angola
}

Undergraduate Course Duration in Medicine:
an Estimation in the 15 Graduate Cohorts at the
Agostinho Neto University, Angola

Pedro Magalhães ${ }^{I}$

Guilherme Bugalho Gomes ${ }^{I}$

Santos Morais Nicolau

\section{PALAVRAS-CHAVE}

- Educação Médica.

- Tempo.

- Angola.

\section{RESUMO}

O tempo de conclusão do curso de graduação em Medicina está bem estabelecido nos programas de formação das escolas médicas em todo o mundo. Entretanto, nem todos os estudantes concluem o curso no tempo esperado, o que pode resultar numa baixa taxa de graduação. Contudo, uma análise isolada da taxa de graduação não permite prever com precisão a magnitude de custos de formação se não levar em conta o tempo médio de conclusão da formação, particularmente para a Medicina, que exige avultados recursos e cujo tempo de formação é o mais longo. O objetivo do estudo foi determinar o tempo médio de permanência no curso, assim como a proporção de estudantes que se gradua no tempo esperado de conclusão do curso. Foi feita uma análise retrospectiva dos dados de 15 coortes de estudantes graduados pela Faculdade de Medicina da Universidade Agostinho Neto (FMUAN), em Angola, no período de 2001 a 2015. Os dados foram recolhidos do arquivo acadêmico da FMUAN. Do total de 1.259 graduados, 59,7\% eram mulheres, e a média de idade na conclusão do curso foi de 35 \pm 7 anos. A taxa média de admissão ao curso foi de 6,8\%, e foram graduados, em média, 88 médicos por ano, sendo 36 homens e 52 mulheres. A taxa de graduação foi de $82,2 \%$, sendo que os estudantes demoraram, em média, dez anos para concluir o curso, e apenas $24,2 \%$ concluíram o curso no tempo esperado de seis anos. Os resultados sugerem que, apesar de ter havido uma elevada taxa de graduação, poucos concluem o curso no tempo regulamentar, realçando a importância de identificar as causas da estadia prolongada de estudantes no curso, o que pode ter implicação na gestão acadêmica e na planificação de recursos humanos de saúde. A taxa de graduados no tempo ideal, combinada com a taxa de graduação, pode ser um indicador de eficiência e um instrumento de apoio na gestão do sistema de educação médica. 


\section{KEYWORDS}

- Medical Education.

- Time.

- Angola

Recebido em: 08/03/2017

Aprovado em: 20/04/2017

\section{ABSTRACT}

The time required to complete undergraduate medical courses is well established in medical school training programs worldwide. However, not all students complete the degree in the expected timeframe, which can lead to a low graduation rate. Nevertheless, an isolated analysis of the graduation rate does not accurately predict the magnitude of training costs if it does not take into account the average completion time, particularly for undergraduate medical course for which the training time is longer and requires substantial resources. The aim of this study was to determine the length of time to complete the degree, as well as the proportion of students who graduated within the regular timeframe. In this retrospective study, we analysed data from 15 cohorts of medical students who finished the undergraduate program at the Faculty of Medicine of the Agostinho Neto University (FMUAN) in Angola, from 2001 to 2015. Data were collected from the academic records registered at the FMUAN. From the total number of graduates ( $n=1259), 59.7 \%$ were women and the average age at course completion was $35 \pm 7$ years. The average admission rate was $6.8 \%$, and it was found that 88 students (36 men and 52 women) had graduated per year. On average, students took 10 years to complete the degree. The graduation rate was $82.2 \%$, and only $24.2 \%$ of the total graduates concluded the program in the regular period of six years. The results suggest that despite a high graduation rate, less than one third of the students finished the training program within the expected timeframe, highlighting the need to identify the causes of prolonged course duration which may have implications for both academic management and for human resource planning in health. Thus, a combined analysis of graduation rate and the time to complete the undergraduate course may be a good indicator of the efficiency of the medical education system.

\section{INTRODUÇÃO}

Em todo o mundo, as escolas médicas enfrentam um cenário comum, caracterizado por um elevado custo da formação de futuros profissionais, num contexto em que a procura é bem maior do que as vagas. Como consequência, há uma intensa disputa por vaga entre os candidatos e baixa capacidade de absorção de novos estudantes.

Geralmente, os programas de formação médica são concebidos para permitir que os estudantes ganhem habilidades e competências profissionais essenciais ao exercício da profissão, com um padrão de qualidade recomendado ${ }^{1}$.Assim, a duração de um curso de graduação é ajustada ao programa de formação, cuja estrutura curricular pode variar entre diferentes países em função das necessidades da sociedade e de suas condições tecnológicas, clínicas e científicas. Contudo, independentemente dos programas de formação médica, uma baixa taxa de graduação em Medicina pode comprometer a execução a longo prazo de programas públicos do Sistema Nacional de Saúde, pois tem implicação direta na planificação da força de trabalho do setor da saúde, que deve contar com os novos médicos que se formam a cada $a^{2,3}$ para o cumprimento efetivo da sua missão.
Apesar de o tempo de conclusão do curso de graduação em Medicina estar bem estabelecido nos programas de formação, nem todos os estudantes concluem o curso no tempo esperado e alguns acabam por desistir. Na base disso, podem estar razões acadêmicas (reprovação e consequente repetição de ano, anulação temporária de matrícula, prescrição acadêmica, mudança de curso) e razões não acadêmicas (fatores sociais, falta de recursos para pagamento de propinas, problemas de saúde, etc.).Em sua maioria, os estudos sobre a formação médica têm centrado sua abordagem nas taxas de graduação e fatores associados ao insucessoacadêmico ${ }^{4-6}$, masmuito pouco tem sido reportado sobre o tempo médio que os estudantes demoram para concluir a sua formação ou sobre a proporção que se forma no tempo esperado de conclusão do curso.

Embora seja um importante indicador de rendimento de um sistema educativo, a taxa de graduação não permite prever com precisão a magnitude de custos de formação se não levar em conta o tempo médio de conclusão de curso, particularmente para a Medicina, que exige avultados recursos e cujo tempo regulamentar de formação é o mais longo em qualquer parte do mundo. Portanto, é importante conhecer o tempo médio que os estudantes demoram para concluir um curso de 
graduação, de modo a permitir uma estimativa realista dos custos de formação por estudante, o que pode ser útil na busca de melhor estratégia que otimize a relação entre o investimento (recursos humanos, materiais, financeiros e tempo) e o produto alcançado (número de graduados).

Com este estudo, pretende-se determinar o tempo médio de graduação, assim comoa proporção de estudantes que conclui o curso nos prazos estabelecidos no programa de formação médica vigente em Angola. Secundariamente, fez-se uma projeção do número de egressos. Deste modo, os resultados deste estudo podem proporcionar subsídios à autoavaliação, servindo como um dos indicadores de eficiência do sistema educativo e de planificação da admissão de novos estudantes ao curso, assim como para a gestão de recursos humanos no Sistema Nacional de Saúde.

\section{METODOLOGIA}

\section{Delineamento e tipo do estudo}

Trata-se de um estudo descritivo observacional em que foram analisados os dados de estudantes graduados em Medicina ao longo de 15 anos na Faculdade de Medicina da Universidade Agostinho Neto (FMUAN), em Angola, no período de 2001 a2015. Os dados foram recolhidos sob anonimato após prévia autorização da Direção da instituição, e as variáveis de interesse foram codificadas. Não foi obtido o consentimento informado dos graduados pela dificuldade de obtenção retrospectiva do mesmo.

\section{Estrutura e funcionamento do curso de Medicina na Universidade Agostinho Neto (UAN)}

A FMUAN é a mais antiga escola pública de formação médica do país, e sua história remonta ao ano de1962. O acesso ao curso é condicionado à existência de vagas previamente definidas pelo Conselho pedagógico de cada Unidade Orgânica ${ }^{7,8}$.

Os estudantes são admitidos ao curso com base nas notas obtidas no exame de acesso ou mediante transferência ${ }^{7}$. O curso tem a duração de seis anos e integra duas grandes componentes (pré-clinica e clínica). Está dividido em três ciclos: básico (primeiro e segundo anos), clínico (terceiro, quarto e quinto anos) e estágio (sexto ano). O ciclo básico integra o ensino sobre ciências básicas. A partir do terceiro ano, o ensino é predominantemente prático, e os estudantes permanecem a maior parte do tempo em hospitais e nas unidades sanitárias da rede primária de saúde, onde fazem rotações supervisionadas para aprender sobre habilidades clínicas, profissionalismo e investigação científica. No ciclo de estágio, os estudantes fazem rotações em diferentes serviços médico-cirúrgicos e de saúde pública, e são integrados em várias equipes médicas ao longo de um ano ${ }^{9,10}$.
A conclusão do curso depende da aprovação em todas as disciplinas nos três ciclos. O ciclo de estágio inclui uma avaliação dos atos clínicos praticados em cada área de rotação e culmina com uma apresentação e defesa pública individual do relatório de um estudo acadêmico-científico ("trabalho de fim de curso") perante um júri designado para esse efeito ${ }^{10}$.

\section{População de estudo, recolha e processamento dos dados}

Os dados foram recolhidos das fichas acadêmicas de cada graduado, e complementados com informações obtidas das pautas de exames de acesso e respectivos relatórios, que se encontram arquivadas no departamento acadêmico da FMUAN. Foi considerado como graduado todo estudante que tenha concluído com sucesso os três ciclos de formação e cuja média final do curso estava registrada na respectiva ficha acadêmica. Ao todo, foram localizadas 1.292 fichas. Para se evitar uma distorção (confusão positiva) dos resultados na estimativa do tempo de duração da formação, foram excluídas 33 fichas referentes a graduados cujo tempo de permanência no curso era de menos de seis anos porque estes ingressaram no curso por via de transferência e consequente integração curricular, à luz do regime acadêmico vigente ${ }^{7,8}$.Assim, os dados analisados se referem aos 1.259 graduados no período em análise.

A taxa de admissão foi expressa em percentagem de candidatos admitidos ao curso em determinado ano em relação ao total de candidatos inscritos ao exame de acesso no mesmo período. A taxa de graduação foi expressa em percentagem de graduados em determinado ano em relação ao total de admitidos no curso no ano de ingresso.

\section{Análise dos dados}

Os dados foram processados numa planilha Excel. Os resultados foram expressos em percentagens e valores absolutos, e descritos como valores médios e medianos.

Foram ensaiados diferentes modelos matemáticos para prever a evolução do número de egressos, de acordo com o método descrito por Ozcan: ${ }^{11}$ os modelos naïf, média móvel simples com três, quatro e seis períodos (MM3, MM4 e MM6, respectivamente); médias ponderadas para os mesmos períodos (MMP3, MMP4 e MMP6); o modelo de alisamento exponencial simples (AES) com coeficiente alfa $=0,3$ e alfa $=0,5$; e o modelo de regressão linear simples.

\section{Seleção do modelo de previsão de egressos}

O número de egressos foi estimado por meio do ensaio de vários modelos matemáticos, tendo-se optado por aquele que se mostrou o mais adequado. Tendo em conta que a análise exploratória dos dados indicava certa tendência, também foi 
ensaiado o modelo de regressão linear simples para modelar tal tendência.

Com base nos valores do erro absoluto médio (EAM) e do erro absoluto percentual médio (EAPM), o modelo de regressão linear pareceu ser mais preciso na previsão da evolução do número de licenciados, seguido do modelo MM3, pois ambos apresentaram valores de EAM e EAPM mais baixos. Entretanto, ao se aferir a consistência dos dois modelos por meio do teste de sinal de controle, o modelo MM3 se mostrou mais consistente por ter apresentado um padrão de variação de sinal mais estável comparativamente ao modelo de regressão linear, sugerindo que um outro modelo não linear poderia permitir melhor previsão. Por essa razão, preferiu-se usar o modelo MM3, que se mostrou o mais robusto para produzir previsões adequadas com menor margem de erro para esta série de dados. Os resultados do AEM e do AEPM para média móvel ponderada (MMP) de diferentes períodos foram: MMP3 - 14.353 e 0,1867, respectivamente; MMP4 - 14.950 e 0,1887, respectivamente; e MMP6 -17.393 e 0,2079, respectivamente.

\section{RESULTADOS}

Do total de 1.259 graduados, mais da metade (59,7\%) eram mulheres. A média de idade na conclusão do curso foi de $35 \pm 7$ anos, sendo $36 \pm 7$ anos para os homens e $35 \pm 7$ anos para as mulheres.

Analisando-se os dados de resumos estatísticos dos exames de acesso referentes ao período de 2001 a 2014 (Figura 1), notou-se que a média anual de vagas para a admissão de novos estudantes foi de 107 (mínimo 80 e máximo 120), e houve, em média, 1.547 candidatos inscritos ao exame de acesso ao curso. A taxa média de admissão foi de $6,8 \%$, tendo sido observado o valor mais elevado no ano de 2002 (11,2\%) e o menor no ano de 2008 (4,5\%). Nesse período, cada vaga era disputada, em média, por 15 candidatos.

Tempo de conclusão do curso e número de formados no período 2001-2015

A Tabela 1 mostra que os estudantes demoraram, em média, dez anos para concluir o curso, independentemente do sexo. No entanto, seis estudantes demoraram 27 anos para concluir, sendo quatro homens e duas mulheres.

Ao longo dos 15 anos, a UAN graduou, em média, 88 médicos por ano, sendo 36 homens e 52mulheres. Ao analisar-sea proporção de estudantes que concluíram sua formação no tempo esperado, os resultados foram surpreendentes. A Figura 2 mostra que, do total de graduados, apenas cerca de um quarto $(24,2 \%)$ concluiu o curso nos seis anos previstos no plano de estudos, enquanto metade $(50 \%)$ concluiu o curso em seis ou oito anos.
Figura 1

Candidatos ao exame de acesso, vagas e taxa de admissão ao curso na FMUAN, período 2001-2014. Os números sobre os pontos representam os valores absolutos de vagas (numerador) em relação ao total de candidatos inscritos nos anos respectivos (denominador)

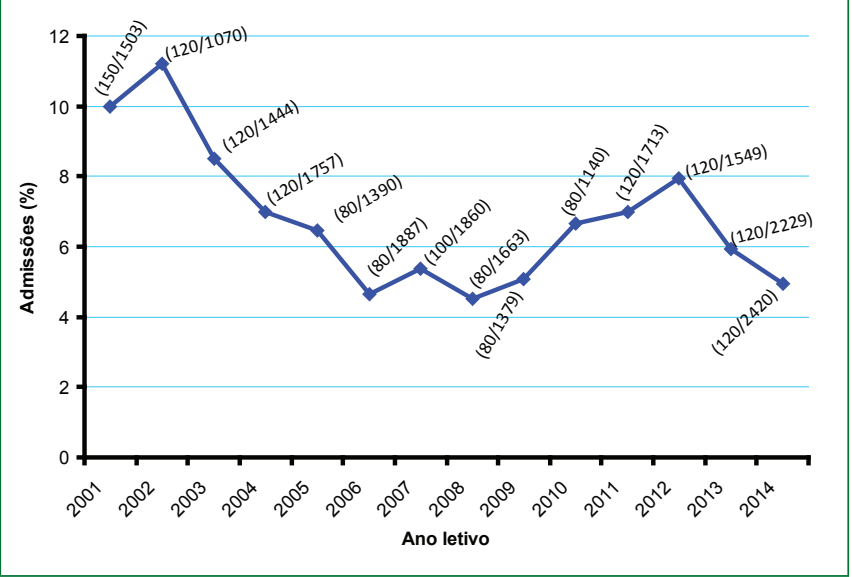

\begin{tabular}{|c|c|c|}
\hline \multicolumn{3}{|c|}{$\begin{array}{l}\text { Tempo de formação em Medicina na } \\
\text { UAN, período 2001-2015 }\end{array}$} \\
\hline & \multicolumn{2}{|c|}{ Tempo de formação (em anos) } \\
\hline & Média \pm DP & Mediana (pc 25-75) \\
\hline Homens $(n=507)$ & $10 \pm 5$ & $8(6-12)$ \\
\hline Mulheres $(n=752)$ & $10 \pm 4$ & $9(7-12)$ \\
\hline Total $(\mathrm{n}=1.259)$ & $10 \pm 4$ & $8(6-12)$ \\
\hline
\end{tabular}

$D P=$ desvio-padrão; $p c=$ percentis.

Figura 2

Frequências acumuladas do tempo de formação em Medicina na UAN, período 2001-2015

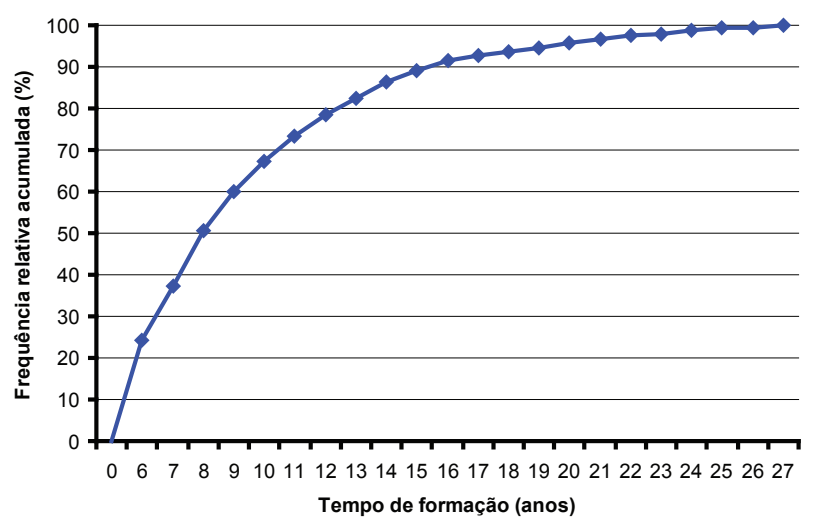


Considerando o número médio de graduados por ano ( $n=88)$ em relação ao número de candidatos admitidos no mesmo período $(n=107)$, isto resulta numa taxa média de graduação de $82,2 \%$.

Evolução do número de graduados e previsão para o período 2015-2019

Como era esperado, os resultados mostram ter havido uma variação do número de graduados ao longo de 15 anos, com uma tendência de incremento do número de egressos, cujo pico foi notório a partir do ano letivo de 2005 (Figura 3). Com base na série de dados de licenciados registrados no período de 2001 a 2014, previu-se o número de médicos a formar, para se ter uma visão antecipada da evolução do sistema e, consequentemente, aferir um índice de desempenho (diferença entre o número previsto e o número observado), assumindo um cenário otimista. Com base nos resultados sugeridos pelo modelo MM3, a FMUAN poderá formar mais de cem médicos por ano no quinquênio 2015-2019, sendo: 108 em 2015, 110 em 2016, 108 em 2017, 109 em 2018 e 110 em 2019.

Figura 3

Evolução do número de graduados em Medicina na UAN e previsão para o período 2015-2019. A linha em azul representa o número de graduados registrados, e em verde, os previstos

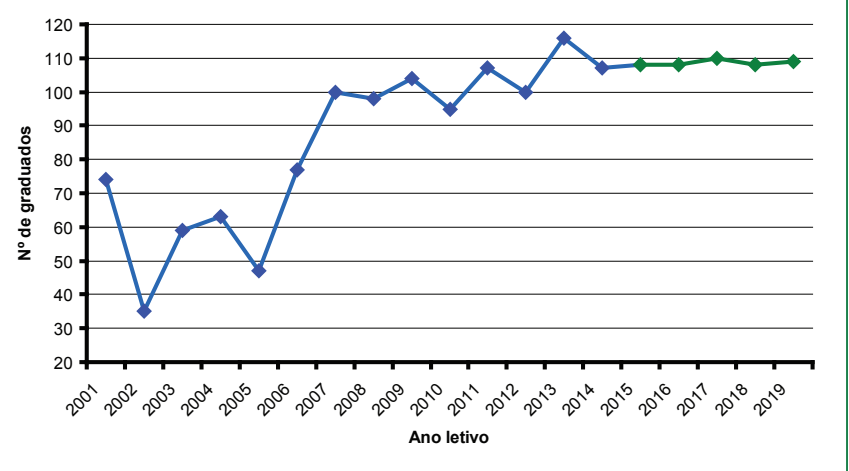

\section{DISCUSSÃO}

Neste estudo, determinou-se o tempo médio de permanência no curso de Medicina, assim como a proporção de graduados que se forma no tempo esperado de conclusão do curso. Para esse efeito, foram analisados os dados acadêmicos referentes a 15 coortes de estudantes graduados numa universidade pública. Os resultados mostram que os estudantes se graduavam ao fim de dez anos, e apenas cerca de um quarto concluía a formação no tempo esperado de seis anos.
Considerando a média de graduados em relação aos admitidos, nota-se que houve uma elevada taxa de graduação $(82,2 \%)$, que se situa na faixa de valores reportados em outras escolas médicas ${ }^{12}$.Contudo, o julgamento deste resultado da taxa de graduação deve ser cauteloso, uma vez que o total de estudantes graduados incluiu tanto os alunos que concluíram o curso ao fim do tempo regulamentar de seis anos, como aqueles que o concluíram fora deste prazo. Esse aspecto é importante para a gestão do sistema de educação médica, porquanto os custos envolvidos na formação podem ser diferentes entre os estudantes que se formam dentro dos prazos em comparação aos que se formam após um período prolongado. A esse respeito, os achados deste estudo mostram um resultado preocupante, porque foi notório que, do total de estudantes que se formaram ao longo de 15 anos, apenas cerca de um quarto se graduou no tempo esperado vigente no país (Figura 2), o que pode ter uma implicação diferente quanto aos custos, assim como na planificação estratégica da força de trabalho.

Embora este estudo não tenha avaliado as causas associadas à variação do tempo de formação, pode-se especular que, entre outros fatores, o baixo rendimento acadêmico dos estudantes pode estar na base disso. Sabe-se que estudantes com baixo rendimento acadêmico acabam por repetir de ano em pelo menos uma ocasião, independentemente dos fatores subjacentes. Em um estudo realizado na Irlanda, Maher e colaboradores ${ }^{13}$ demonstraram que, entre os estudantes de Medicina que desistiam do curso por questões acadêmicas, 48,5\% tinham repetido de ano pelo menos uma vez. Resultados similares foram reportados em outro estudo realizado com estudantes de Medicina de três países africanos lusófonos no ano letivo 2007/2008. Entre outros achados, os pesquisadores observaram que, além de uma frequência considerável debaixo rendimento acadêmico, $12,4 \%, 5 \%$ e $20 \%$ do total de estudantes de Angola, Moçambique e Guiné-Bissau, respectivamente, tinham deixado pelo menos uma disciplina em atraso, principalmente por questões acadêmicas ${ }^{14}$.

Assim, pode ser que a maioria dos graduados que teve o tempo de formação prolongado em nosso estudo também tenha repetido de ano, o que aponta a necessidade de encontrar mecanismos que permitam melhorar o rendimento acadêmico dos estudantes. O tempo de formação prolongado no curso de Medicina pode ser desgastante para o próprio estudante e torna-se mais dispendioso para os governos, sobretudo nos países em que o Estado suporta os custos da formação. Por outro lado, isso impede a criação de vagas para admissão de novos estudantes e pode incrementar o custo de oportunidade para o Estado, em consequência do curto tempo de serviço que os 
futuros profissionais formados ao fim de um longo período de graduação poderão prestar à sociedade.

$\mathrm{O}$ fato de a maioria dos graduados em nosso estudo ter demorado cerca do dobro do tempo de formação estabelecido reforça a ideia de que estes estudantes podem ter repetido pelo menos um ano curricular ao longo da formação. Entre outros aspectos, o regulamento acadêmico da UAN estabelece o "regime de prescrição", que define os termos que condicionam a exclusão definitiva de estudantes que sejam reprovados duas vezes no mesmo ano curricular (no ciclo básico) ou três vezes (no ciclo clínico) ${ }^{78}$. Com base nesse regime, o estudante declarado como prescrito tem o direito auma inscrição suplementar no ano letivo seguinte, durante o qual deverá realizar o último exame que condiciona a sua saída da prescrição ou ser excluído definitivamente do curso. ${ }^{78}$ Deste modo, parece justificar-se que a maioria dos graduado sem nosso estudo tenha concluído o curso ao fim de nove a dez anos(Figura 2), segundo as reprovações tenham ocorrido no ciclo básico ou no ciclo clínico, respectivamente. Por fim, embora a nossa análise não tenha permitido objetivar esse dado, não se deve descurar a possibilidade de ter havido falhas na aplicação efetiva do "regime de prescrição", o que também pode ter contribuído para que alguns dos nossos graduados concluíssem o curso com um tempo excessivamente discrepante em relação à maioria.

Em geral, as escolas médicas são permanentemente desafiadas a admitir mais estudantes e a manter taxas elevadas de graduação, e tudo isso deve ser equacionado com a disponibilidade de recursos adequados para que cumpram a sua missão com eficiência. À semelhança de outros países da África subsaariana, em Angola nota-se que nos últimos anos foi feito um grande esforço para melhorar a qualidade no ensino superior e, em consequência, formar profissionais competentes no país. $\mathrm{O}$ crescimento do número de escolas médicas e o investimento na qualificação e diferenciação acadêmica dos docentes e investigadores são exemplos notórios. Em todo o mundo, as universidades são confrontadas com um número reduzido de vagas para admitir novos estudantes devido às limitações impostas pelo numerus clausus, que é o mecanismo usado para ajustar o ingresso aos cursos superiores em função da disponibilidade de recursos adequados ${ }^{15}$

Na UAN, a admissão de novos estudantes ao curso de Medicina tem sido condicionada à definição prévia do numerus clausus pelo conselho pedagógico ${ }^{9,16}$.Esse mecanismo permite preestabelecer o limite máximo de candidatos que devem ser admitidos ao curso a cada ano em função das condições disponíveis (recursos humanos, materiais, didáticos, etc.), e as vagas devem ser preenchidas pelos candidatos que tenham obtido as melhores classificações no exame de acesso competitivo. Os dados deste estudo mostram que, apesar de ter havido uma variação no número de vagas, este tendia a se elevar e estabilizar. Um padrão de evolução similar também foi observado no número de graduados anualmente e prevê-se uma estabilização em pelo menos cem graduados até o ano de 2019 (Figura 3).Tendo em conta que em Angola persiste ainda um número insuficiente de médicos - 1 médico/10.000 habitantes ${ }^{17}$-, é imprescindível melhorar cada vez mais as condições que permitam aumentar o número de médicos formados na UAN e em outras escolas médicas do país.

Assim, a melhoria das condições de ensino e aprendizagem e o ajustamento dos recursos (financeiros, humanos e materiais) são indispensáveis, já que persiste maior procura por vagas no curso de Medicina em todas as escolas médicas do país, em particular na UAN. Neste sentido, nossos resultados mostram que houve uma intensa disputa por vagas entre os candidatos, a qual, porém, não foi acompanhada de um incremento proporcional da oferta.

Apesar disso, os achados deste estudo não diferem muito dos resultados de um estudo realizado em universidades do Reino Unido no qual foi observado que, do total de 81.422 candidatos ao curso de Medicina, apenas 10\% $(n=8.000)$ tinham sido admitidos ${ }^{18}$.Nesse mesmo período, Universidade de Nottingham havia admitido apenas $9 \%(n=270)$ do total de candidatos inscritos ao teste de admissão $(n=2.500)$ ao curso de Medicina naquela instituição. Assim, embora tenha sido observada menor proporção de candidatos admitidos ao curso na UAN $(6,8 \%)$ relativamente às escolas homólogas do Reino Unido no período em comparação, houve maior disputa por vaga na UAN (15 candidatos por vaga) do que naquelas universidades (cerca de dez candidatos por vaga), possivelmente porque existem mais escolas médicas naquele pais do que em Angola e por suas necessidades de formar médicos serem diferentes, o que ilustra diferentes níveis de desafio para se admitirem novos estudantes anualmente em diferentes escolas. Mesmo assim, uma procura maior pelo curso de Medicina na UAN poderá se manter nos próximos tempos, uma vez que, segundo os dados do censo populacional em 2014, cerca de 6,4\% da população de Angola é constituída por jovens que concluíram o ensino médio, e metade destes $(50,2 \%)$ reside em Luanda ${ }^{19}$, sugerindo que, provavelmente, muitos destes são aspirantes ao curso de Medicina.

\section{Limitação}

Embora o estudo tenha incluído os dados de várias coortes de graduados na mais antiga escola pública de formação médica do País, os resultados não devem ser generalizados a outras 
escolas de Medicina no mundo, porquanto a estrutura do curso, assim como os processos de admissão de estudantes diferem entre países.

\section{CONCLUSÕES}

Os resultados deste estudo sugerem que, apesar de observada uma elevada taxa de graduação, poucos estudantes concluem o curso no tempo esperado de formação, sendo que dois terços concluem o curso em dez anos (o que corresponde a 167\% do tempo regulamentar). Isto poderá implicar custos adicionais de formação e maior dificuldade na gestão da admissão de novos estudantes e na planificação anual do número de médicos necessários para fazer frente aos problemas de saúde da população. Juntos, os resultados sugerem a necessidade de ajustes na gestão acadêmica e a tomada de medidas que visem melhorar o rendimento acadêmico dos estudantes, assim como a necessidade de ajuste da política de admissão ao curso, que permite a seleção dos melhores estudantes.

Estudos longitudinais são promissores para aferir os principais fatores determinantes do tempo prolongado de formação, a fim de que se adotem medidas preventivas, visto que esses fatores podem variar em diferentes contextos e até num mesmo país. Além da análise da taxa de graduação do curso, a determinação da proporção de estudantes que se formam no tempo regulamentar pode ser um indicador de eficiência do sistema de educação médica no nível da pré-graduação.

\section{AGRADECIMENTOS}

Aos funcionários do Departamento acadêmico da FMUAN, pela colaboração na recolha de dados.

\section{REFERÊNCIAS}

1. World Federation for Medical Education - Basic Medical Education WFME Global Standards for Quality Improvement. The 2015 Revision WFME Office Ferney-Voltaire, France Copenhagen, Denmark 2015; [capturado 19 ago. 2016]. Disponível em: http://wfme.org/standards/ bme/78-new-version-2012-quality-improvement-in-basic-medical-education-english/file.

2. Arulampalam W, Naylor R, Smith J: A hazard model of the probability of medical school drop-out in the UK. J R Stat Soc 2004;167:157-178.

3. Imison C, Buchan J, Xavier S. NHS Workforce Planning. Limitations and possibilities. The Kings Fund; 2009. http:// www.kingsfund.org.uk/publications/nhs_workforce.html.

4. Simpson KH, Budd K. Medical student attrition: a 10-year survey in one medical school. Med Educ 1996;30(3):172178.
5. O’Neill LD, Wallstedt B, Eika B, Hartvigsen J. Factors associated with dropout in medical education: a literature review. Med Educ 2011;45:440-454.

6. Yates J. When did they leave and why? A retrospective case study of attrition on the Nottingham undergraduate medical course. BMC Med Educ 2012;12:43.

7. Regime Académico da Universidade Agostinho Neto. Diário da República: II Série, № 21, de 26 de Maio de 2000.

8. Regulamento sobre o Regime Académico da Faculdade de Medicina da Universidade Agostinho Neto, 2002; [capturado 01 set. 2016]. Disponível em: http:/ / www.fmuan.ao/ documentos/regacad.pdf.

9. Regulamento das Estruturas Pedagógicas da Faculdade de Medicina da universidade Agostinho Neto, 2002; [capturado 01 set. 2016]. Disponível em: http:/ / www.fmuan.ao/ documentos/regestacad.pdf.

10. Regulamento do Estágio da Faculdade de Medicina da Universidade Agostinho Neto, 2002; [capturado 01 set. 2016]. Disponível em: http://www.fmuan.ao/documentos/regestagio.pdf.

11. Ozcan YA.Forecasting. In: Quantitative methods in health care management: techniques and applications.2.ed. San Francisco: Jossey-Bass; 2009. p.13-49.

12. Caulfield M, Redden G, Sondheimer H.Analysis in brief: Graduation rates and attrition factors for U.S. Medical School Students. Ass Am Med Coll 2014(14):5.

13. Maher BM, Hynes H, Sweeney C, KhashanAS, O'Rourke M, Doran K, et al. Medical school attrition-beyond the statistics a ten year retrospective study. BMC Med Educ. 2013;13:13.

14. Ferrinho P, Sidat M, Fresta MJ, Rodrigues A, Fronteira I, Silva F, Mercer H, Cabral J, Dussault G. The training and professional expectations of medical students in Angola, Guinea-Bissau and Mozambique [online]. Human Resources for Health 2011. 9 [capturado 28 ago. 2016]; 9. Disponível em: http:/ / www.human-resources-health.com/content/9/1/9

15. Joseph $W$. The numerus clausus and its consequences. Acta Chir Belg. 2007;107(5):493.

16. Simões C. Evolução do "Numerus Clausus" na Faculdade de Medicina da Universidade Agostinho Neto. Rev Angolana Educ Med. 2004; 1(2): 7-13.

17. World Health Organization. African Health Observatory, 2016. Angola: Factsheets of Health Statistics 2016. [capturado 01 set. 2016]. Disponível em: http://www.aho.afro. who.int/profiles_information/images/b/ba/Angola-Statistical_Factsheet.pdf.

18. Universities and Colleges Admission Service. Online statistics 2010; [capturado 8 jul. 2016]. Disponível em: http:/ / 
www.ucas.ac.uk/about_us/stat_services/stats_online/ data_tables/subject/2010.

19. Instituto Nacional de Estatística, 2016. Censo 2014: Resultados Definitivos do Recenseamento geral da População e Habitação de Angola 2014, Luanda. [capturado 04 set. 2016]. Disponível em: http://aiangola.com/wp,content/ uploads /2016/03/Publica\%C3\%A7\%C3\%A3o,Resultado s,Definitivos,Censo,Geral,2014_Vers\%C3\%A3o,22032016_ DEFINITIVA,18H17.pdf.

\section{CONTRIBUIÇÃO DOS AUTORES}

Pedro Magalhães, supervisionou a recolha de dados, fez a análise dos dados e redigiu o manuscrito; Guilherme Bugalho Gomes, fez a recolha dos dados e contribuiu na análise e Santos Morais Nicolau, coordenou o estudo e revisou o manuscrito.

\section{CONFLITO DE INTERESSES}

Os autores declaram não existir conflito de interesses.

\section{ENDEREÇO PARA CORRESPONDÊNCIA}

Pedro Magalhães

Agostinho Neto Medicine School - Physiological Sciences Av. Hoji Ya Henda - Hospital Américo Boavida Luanda 116 - Angola

E-mail: pedromagalhaes24@hotmail.com 